\title{
REVIEW
}

\section{Hiding in plain view: the potential for commonly used drugs to reduce breast cancer mortality}

Michelle D Holmes ${ }^{* 1,2}$ and Wendy Y Chen ${ }^{1,3}$

\begin{abstract}
Many medications have been developed for one purpose but then are found to have other clinical activities. There is tremendous interest in whether non-cancer medications may potentially have effects on breast cancer survival. In this review article, we have presented and evaluated the evidence for several commonly used over-the-counter and prescription medications - including aspirin (and other nonsteroidal anti-inflammatory drugs), beta-blockers, angiotensin-converting enzyme inhibitors, statins, digoxin, and metformin - that have been evaluated among breast cancer survivors in prospective studies. Substantial scientific evidence supports the hypothesis that some of these common and relatively safe drugs may reduce breast cancer mortality among those with the disease by an amount that rivals the mortality reduction gained by currently used therapies. In particular, the evidence is strongest for aspirin (approximately 50\% reduction), statins (approximately $25 \%$ reduction), and metformin (approximately $50 \%$ reduction). As these drugs are generic and inexpensive, there is little incentive for the pharmaceutical industry to fund the randomized trials that would show their effectiveness definitively. We advocate that confirmation of these findings in randomized trials be considered a high research priority, as the potential impact on human lives saved could be immense.
\end{abstract}

\section{Introduction}

Many medications have been developed for one purpose but then are found to have other clinical activities. For example, minoxidil was originally developed as an antihypertensive but then was found to cause excessive hair

\footnotetext{
*Correspondence: michelle.holmes@channing.harvard.edu

The Channing Division of Network Medicine, Department of Medicine, Brigham and Women's Hospital and Harvard Medical School, 181 Longwood Avenue,

Boston, MA 02115, USA

Full list of author information is available at the end of the article
}

growth. Because of the multiple potential pathways that can be involved with cancer growth and metastases, tremendous interest remains in whether currently used non-cancer medications may potentially have anti-cancer effects. In this review article, we will present and evaluate the evidence for several commonly used over-thecounter and prescription medications that have been evaluated among breast cancer survivors in prospective studies. Please note that we have not included a discussion of selective serotonin reuptake inhibitors and tamoxifen, since this appears to be more of a pharmacologic interaction rather than a true anti-cancer effect. We have focused our discussion on drugs that may influence cancer recurrence rather than primary incidence.

\section{Methods}

For this review article, we will focus on cohort studies, prospective nested case control studies, and randomized controlled trials that presented breast cancer-specific survival or recurrence data. We have omitted case control studies because these can be subject to bias. For our search strategy, we searched PubMed through July 2012 for relevant English language studies. The major search terms used were breast neoplasms and (mortality or survival or survival analysis or survivors or recurrence). For the individual drug search terms, we used (aspirin or anti-inflammatory agents, non-steroidal), adrenergic betaantagonists, (angiotensin-converting enzyme inhibitors or angiotensin receptor antagonists), (insulin or hypoglycemic or metformin), (statin or lovastatin or simvastatin or fluvastatin or atorvastatin or pravastatin or rosuvastatin), and (digoxin or digitalis). We also reviewed the references lists of all relevant papers for any additional studies. We did not include studies that were presented only in abstract form at a meeting or were published only as editorial letters.

\section{Aspirin and other non-steroidal anti-inflammatory drugs \\ Biological rationale/preclinical data}

Aspirin and non-steroidal anti-inflammatory drugs (NSAIDs) may influence breast cancer recurrence through a number of mechanisms. They inhibit production of 
prostaglandins and cyclo-oxygenase (COX), which comes in two isoforms: COX-1 and COX-2 [1]. It has been known for over 20 years that elevated tissue levels of prostaglandins have been seen in breast tissue, especially hormone receptor-negative tumors [2]. Prostaglandins can stimulate angiogenesis [3] and inhibit apoptosis [4]. In addition, prostaglandins stimulate aromatase activity and thus may affect estrogen production [5]. Aromatase is an enzyme that catalyzes the conversion of androgen precursors to estrogen, the main source of estrogen production in post-menopausal women. Aromatase inhibitors are widely used for breast cancer treatment and lower estrogen levels. Aspirin and NSAIDs could improve survival if they acted as aromatase inhibitors. Cross-sectional studies provide suggestive evidence that aspirin can influence estrogen levels, since estrogen levels are lower among women using aspirin [6]. However, prostaglandin effects may not be limited to hormone receptor-positive tumors.

There is also strong evidence that aspirin and NSAIDs may prevent early metastasis but not advanced disease. COX-2 overexpression has been associated with human breast cancer that has metastasized [7]. This may explain why early trials of NSAIDs to treat advanced or metastatic breast cancer showed little effect [8]. A recent publication reviewed the extensive experimental evidence showing that platelets promote adhesion of circulating tumor cells to the endothelium and protect them from immune elimination within the circulatory system, thus enabling future establishment of metastases. Aspirin, but not NSAIDs, inhibits platelet function [9].

\section{Epidemiologic data}

Three out of four large prospective observational studies have shown a potential survival benefit among women with breast cancer who use aspirin or NSAIDs. Kwan and colleagues [10] reported from the Life After Cancer Epidemiology (LACE) study, a prospective cohort of 2,292 survivors whose stage I to III breast cancer was diagnosed between 1997 and 2000 and who were drawn primarily from Kaiser Permanente Northern California. The authors found a reduced risk of recurrence for current regular ( $>3$ days per week) use of ibuprofen (relative risk $(\mathrm{RR})=0.56,95 \%$ confidence interval $(\mathrm{CI})=$ 0.32 to 0.98 ) but not aspirin ( $R R=1.09,95 \% \mathrm{CI}=0.74$ to 1.61). However, short follow-up (mean of 2.5 years) may have precluded the detection of an association. Blair and colleagues [11] reported a borderline reduced risk of breast cancer death ( $R R=0.64,95 \% \mathrm{CI}=0.39$ to 1.05 ) for any use of NSAIDs (aspirin and non-aspirin NSAIDs combined) after diagnosis among 591 post-menopausal women with breast cancer and a reduced risk of breast cancer death for aspirin use alone $(\mathrm{RR}=0.53,95 \% \mathrm{CI}=$ 0.30 to 0.93$)$. In the combined group of any NSAID use, use of aspirin only (43\%) was considerably more common than use of non-aspirin NSAIDs only $(10 \%)$ or use of both (27\%). In the Nurses' Health Study [12], we reported on 4,164 women with early-stage breast cancer and found a reduced risk of breast cancer death for aspirin use after diagnosis $(\mathrm{RR}=0.51,95 \% \mathrm{CI}=0.41$ to 0.65$)$. The survival benefit was similar for estrogen receptor (ER)-positive and -negative tumors. There was a suggestion of a protective association with NSAID intake as well, but power was limited. Intriguingly, among a subset of 2,001 subjects for whom we had tumor samples to perform COX-2 immunohistochemistry, we found a similar association for aspirin use among those with COX-2positive tumors ( $R R=0.64,95 \% \mathrm{CI}=0.43$ to 0.96$)$ and COX-2-negative tumors $(\mathrm{RR}=0.57,95 \% \mathrm{CI}=0.44$ to 0.74 ), suggesting that the aspirin mechanism for breast cancer may be independent of COX-2 [7]. Aspirin binds covalently to and inhibits both COX-1 and COX-2. In breast carcinogenesis, in contrast to colon cancer, COX-1 activity may be relatively more important [13].

Among 1,024 breast cancer cases from a populationbased case control study followed as a cohort for an average of 7 years, Li and colleagues [14] reported a nonstatistically significant reduced risk of overall mortality among those using aspirin $(\mathrm{RR}=0.82,95 \% \mathrm{CI}=0.54$ to $1.24)$ and a similar risk for breast cancer mortality $(\mathrm{RR}=$ $0.89,95 \% \mathrm{CI}=0.53$ to 1.52 ).

In addition to the prospective studies, randomized trial data have demonstrated an effect of aspirin on cancer recurrence. In the UK, Rothwell and colleagues [15] pooled data from five large randomized trials of aspirin to prevent vascular disease. The purpose of the pooled analysis was to examine the effect of aspirin on cancer metastases presenting during or after the trials' followup. In the pooled data, those subjects allocated to aspirin had a reduced risk of cancer with distant metastasis, mainly due to a reduced risk of metastatic adenocarcinoma $(\mathrm{RR}=0.52,95 \% \mathrm{CI}=0.35$ to 0.75$)$. In addition, patients with adenocarcinoma who did not have metastasis at initial diagnosis and who remained on aspirin up to or after diagnosis had a markedly reduced risk of metastasis during follow-up $(\mathrm{RR}=0.31,95 \% \mathrm{CI}=$ 0.15 to 0.76 ). Examination of case fatality by individual cancers was hampered by small numbers, but there was a suggestion of reduced case fatality for breast cancer $(\mathrm{RR}=0.16,95 \% \mathrm{CI}=0.02$ to 1.19$)$. Because these dramatic pooled findings were similar in the one trial which used a low-dose $(75 \mathrm{mg})$ slow-release formulation of aspirin designed to inhibit platelet function only in the portal circulation and not to have systemic effects, the authors speculate that aspirin's effect on platelet-mediated formation of metastases is the likely mechanism [15].

Corroboration was provided by a linked meta-analysis comparing data from observational studies with those 
from the randomized trials. The risk of breast cancer with distant metastases pooled from observational studies ( $R R=0.58,95 \% \mathrm{CI}=0.20$ to 1.71 ) was similar to that found in randomized trials but, owing to small numbers, did not reach statistical significance [16].

In conclusion, abundant preclinical and epidemiologic data support a protective role for aspirin and NSAIDs in breast cancer survival [10-12,16]. In addition, pooled data from five large randomized trials of aspirin used to prevent vascular disease have demonstrated a reduced risk of metastatic and fatal adenocarcinoma, including breast cancer, among those allocated to aspirin. Results from the randomized trials hint that low-dose $(75 \mathrm{mg})$ aspirin may be effective, suggesting that inhibition of platelet function may be the key mechanism in preventing metastases [15]. However, as we reported in a previous review, several other mechanisms may be involved for breast cancer [6]. Although COX-2 expression is strongly linked to the cancer process, for breast cancer these beneficial effects are not solely or primarily caused by inhibiting COX-2 [7]. Although nonaspirin NSAIDs may also improve breast cancer survival, evidence is currently strongest for aspirin $[13,15]$.

\section{Beta-blockers}

\section{Biological rationale/preclinical data}

Patients and clinicians have shown great interest in the possible link between stress and cancer initiation and progression. In a 2006 review, Antoni and colleagues [17] elucidated how bio-behavioral influences (for example, life stress, psychological processes, and health behaviors) could plausibly affect cancer processes through neuroendocrine pathways. In fact, evidence is stronger for an effect on cancer progression than on cancer initiation [17].

The major neuroendocrine transmitters of the stress response are catecholamines, and beta-adrenergic receptors mediate most of the effects of catecholamines. Preclinical studies in several types of cancer (ovarian, nasopharyngeal, prostate, and pancreatic) have shown catecholamine stimulation to increase angiogenesis, tumor invasion, metastasis, and inhibit apoptosis; many of these effects could be inhibited by the use of betaadrendergic blocker drugs such as propranolol [18].

In a recently published study, mice with mammary cancer subjected to chronic stress had neuroendocrine activation that did not affect growth of the primary tumors but increased distant metastases 30-fold. This tumor spread could be inhibited by treatment with propranolol [19]. Beta-adrenergic receptors have been found in human breast cancer cells [20].

\section{Epidemiologic data}

Four observational studies among women with breast cancer (three cohorts and one prospective nested case control) have examined the association between intake of beta-blockers and risk of either breast cancer mortality or recurrence. In 2010, Powe and colleagues [21] reported on 466 stage I to III UK breast cancer patients with more than 10 years of follow-up; 92 (20\%) had pre-existing hypertension and 43 of these (9\%) were treated with betablockers. In multivariate models controlling for age and tumor characteristics, women using beta-blockers had marked decreases in breast cancer mortality $(R R=0.29$, $95 \% \mathrm{CI}=0.12$ to 0.72$)$ and distant recurrence $(\mathrm{RR}=0.43$, $95 \% \mathrm{CI}=0.20$ to 0.93 ) [21]. This initial small study was rapidly followed by three larger ones published in 2011 . Melhem-Bertrandt and colleagues [22] reported on 1,413 patients with stage I to III breast cancer at the MD Anderson Cancer Center; 102 (7\%) used beta-blockers. The authors hypothesized that the higher prevalence of abdominal obesity and metabolic syndrome among women with triple-negative breast cancer and its link to adrenergic dysregulation and also high expression of beta-adrenergic receptors in triple-negative breast cancer cell lines could make these patients particularly sensitive to beta-blocker treatment. The authors reported a decreased risk of relapse $(\mathrm{RR}=0.52,95 \% \mathrm{CI}=0.31$ to 0.88 ) for users of beta-blockers among all patients. This was most pronounced among the 377 patients with triple-negative breast cancer $(\mathrm{RR}=0.30,95 \% \mathrm{CI}=0.10$ to 0.87 ) [22]. Ganz and colleagues [23] reported on 1,779 women with stage I to IIIA breast cancer from the LACE cohort, all of whom had linked pharmacy records. Mean follow-up was 8.2 years, and 270 of the women (15\%) used beta-blockers. The authors found non-statistically significant decreased risks of breast cancer death $(\mathrm{RR}=$ $0.76,95 \% \mathrm{CI}=0.44$ to 1.33 ) and recurrence (distant, locoregional, or contralateral, $\mathrm{RR}=0.85,95 \% \mathrm{CI}=0.57$ to 1.32) among users of beta-blockers [23]. The fourth study is a nested case control reported by Barron and colleagues [24] linking the Irish national cancer registry and pharmacy registries. They studied 5,333 women with stage I to IV breast cancer. Five hundred ninety-five betablocker users (70 using propranolol and 525 using atenolol) were matched 1:2 on factors associated with breast cancer screening and other healthy behaviors (including socioeconomic status, smoking, aspirin, and statin use) to controls not using a beta-blocker. The 70 propranolol users had a markedly decreased risk of breast cancer mortality $(\mathrm{RR}=0.19,95 \% \mathrm{CI}=0.06$ to 0.60 ) compared with non-beta-blocker users, but this was based on only four breast cancer deaths among propranolol users. Propranolol users also were less likely to present with locally advanced or metastatic tumors. No such association was seen for atenolol [24]. Propranolol is non-selective and blocks both beta- 1 and beta- 2 adrenergic receptors, whereas atenolol blocks only beta-1. Historically, over time, patterns of use have 
moved from the non-selective to the cardioselective (beta-1) blockers. The authors present preclinical evidence that beta- 2 signaling may be more important for cancer metastasis, and their results would seem to bolster this hypothesis [24].

In conclusion, tantalizing evidence from preclinical studies supports a role for beta-blockers to inhibit breast cancer metastasis and potentially improve survival. Observational studies are hampered by a relatively low prevalence (approximately $10 \%$ to $15 \%$ ) of beta-blocker use and the fact that only a subset of non-selective betablockers may be effective, and these have been used less over time. Additionally, the beta-blocker effect may differ by tumor subtype, with a stronger effect seen among triple-negative tumors.

\section{Angiotensin-converting enzyme inhibitors and angiotension type I receptor blockers \\ Biological rationale/preclinical data}

The renin-angiotensin-aldosterone system (RAAS) has a potential role in breast cancer control. Angiotensin I is cleaved into angiotensin II by angiotensin-converting enzyme (ACE). Angiotensin II interacts with angiotensin type I receptors to promote aldosterone secretion and vasoconstriction. ACE inhibitors (ACEIs) and angiotensin type I receptor blockers (ARBs) are commonly used to treat hypertension, congestive heart failure, and chronic kidney disease [18].

Polymorphisms of the RAAS genes leading to increased activity of the system have been associated with increased risk of breast cancer [25,26]. Breast cancer cells have been found to express components of the RAAS [27]. RAAS stimulation of breast cancer cells can increase cell proliferation via protein kinase $\mathrm{C}$ activation and epidermal growth factor receptor transactivation as well as activating the P13K-kinase B (AKT) pathway [28,29]. RAAS stimulation of hormone receptor-negative breast cancer cells has been shown to increase expression of angiogenesis-related genes [27].

\section{Epidemiologic data}

Two observational studies that previously reported on use of beta-blockers and breast cancer survival also reported on ACEI/ARB use. Contrary to the hypotheses generated by the preclinical evidence, neither the MD Anderson cohort of 1,413 patients reported by MelhemBertrandt and colleagues [22] nor the LACE cohort $(\mathrm{n}=$ 1,779) reported by Ganz and colleagues [23] found any evidence of decreased recurrence, breast cancer mortality, or total mortality among women with breast cancer using ACEIs or ARBs (Table 1) [22,23]. In fact, an elevated risk of recurrence was found among the LACE cohort $(\mathrm{RR}=1.56,95 \% \mathrm{CI}=1.02$ to 2.39$)$ [23]. In a smaller cohort of 703 stage II/III breast cancer patients from Albert Einstein Medical Center, Chae and colleagues [30] reported a reduced risk of breast cancer recurrence among those using $\mathrm{ACEI} / \mathrm{ARB}(\mathrm{RR}=0.49$, $95 \% \mathrm{CI}=0.31$ to 0.76 ), but total mortality was not reduced. Therefore, despite promising preclinical evidence for ACEIs/ARBs, substantial evidence for a protective effect among women with breast cancer is currently lacking.

\section{Statins}

\section{Biological rationale/preclinical data}

Statins - HMG-CoA (3-hydroxy-3-methyl-glutaryl-CoA) reductase inhibitors - are widely used lipid-lowering drugs. Interestingly, lipophilic statins (for example, simvistatin, lovastatin, and fluvastatin) have been shown in vitro to inhibit breast cancer cell growth and proliferation with a variety of hypothesized mechanisms. In multiple cell lines, statins can inhibit prenylation (post-translational modification) of multiple proteins, including those in the Ras family, which is involved in signal transduction and presumed to be important in carcinogenesis [31]. Statins may also inhibit histone deacetylase activity [32]. Drugs targeting histone deacetylation are already approved for lymphoma and have activity in other cancers as well. Several clinical trials in cancers other than breast cancer have suggested that statins used in conjunction with chemotherapy may improve efficacy [18]. In terms of breast cancer incidence, studies on the effects of statins are mixed. However, the only published, cohort studies on the association between statins and breast cancer recurrence have consistently shown a decreased risk of recurrence.

\section{Epidemiologic data}

The first published study used the LACE population. Statin use was confirmed via pharmacy records, and health outcomes were verified by questionnaire and medical record review. Use of statins for more than 100 days after diagnosis compared with shorter-term use was associated with a non-significant decreased risk of cancer recurrence $(R R=0.67,95 \% C I=0.39$ to 1.13$)$ after adjustment for age at diagnosis, race, body mass index (BMI), cancer stage, and tamoxifen use. Breast cancer recurrence risk decreased with increasing duration of post-diagnosis statin use $(P$ for trend $=0.02)$. However, power was limited, as there were only 16 recurrences among survivors who used statins more than 100 days after diagnosis. The primary statin used in this cohort was the lipophilic lovastatin, which accounted for $84 \%$ of statin use among regular statin users [33]. The study by Chae and colleagues [30], which was previously cited on ACEI/ARB, also evaluated the association with statin use and reported a decrease risk of recurrence (multivariate hazard ratio $(\mathrm{HR})=0.40,95 \% \mathrm{CI}=0.24$ to 0.67 ) and no 
Table 1. Cohort studies of aspirin, beta-blockers, angiontensin-converting enzyme inhibitors, statins, and metformin and breast cancer survival

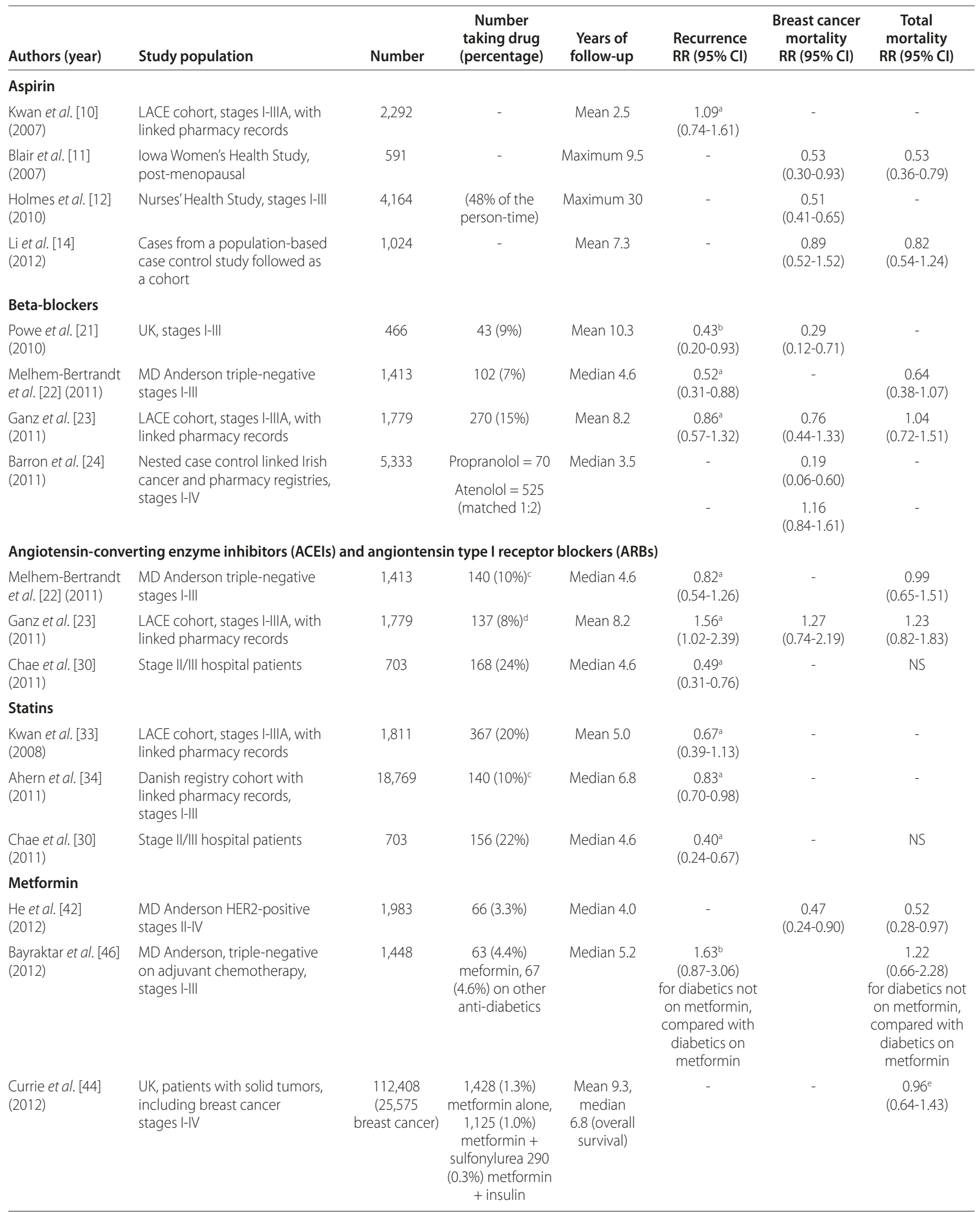

aAny recurrence; ${ }^{b}$ distant recurrence; ${ }^{\mathrm{A}} \mathrm{ACEl}$ or ARB use; ${ }^{\mathrm{d}} \mathrm{ACEl}$ only; ${ }^{\mathrm{e}}$ breast cancer survivors only; see [44] for all cancer survivor numbers. Cl, confidence interval; $\mathrm{LACE}$, Life After Cancer Epidemiology; NS, not significant; RR, relative risk. 
impact on overall survival. Power was also limited in this study, as there were only 19 recurrences among those who used statins at least 6 months [30].

The largest study to date was a population-based Danish cohort of 18,769 survivors of stage I to III breast cancer diagnosed between 1996 and 2003. Statin use was assessed via linkage to the National Registry of Medicinal Products, which tracks all prescriptions since 1995. Breast cancer recurrence was confirmed via cancer registry data. Women who used primarily lipophilic statins had a lower risk of recurrence compared with non-users (10-year $\mathrm{HR}=0.73,95 \% \mathrm{CI}=0.60$ to 0.89$)$ after adjustment for age at diagnosis, menopausal status, cancer stage, ER status, cancer treatment, and use of other relevant non-prescription medications. In contrast, women who used primarily hydrophilic statins (for example, atorvastatin, pravastatin, or rosuvastatin) had the same risk of breast cancer recurrence as non-users (10-year adjusted HR $=1.2,95 \% \mathrm{CI}=0.79$ to 1.70$)$. It should be noted that the analyses with hydrophilic statins were limited for power; there were only 39 recurrences at 10 years compared with 182 recurrences among users of lipophilic statins. The primary statin used in the Danish cohort was simvastatin (accounting for $72 \%$ of prescriptions among statin users), which is the most lipophilic statin. Stratified models showed no difference by grade, ER status, or type of treatment [34]. In the US, prior to the introduction of the generic statins in 2006, Lipitor (atorvastatin; Pfizer Inc, New York, NY, USA) had the largest market share [35].

Although the pharmacologic differences between lipophilic and hydrophilic statins in terms of their cholesterollowering effects have been well characterized, less is known about statins' pleiotropic effects. Hydrophilic, but not lipophilic, statins may increase mevalonate synthesis in extra-hepatic tissues and this may result in differential effects on cancer development. Lipophilic statins also tend to accumulate more in fat and have higher plasma protein binding than hydrophilic statins, and this could result in more extra-hepatic activity and systemic effects [36].

In summary, the observational data on statins influencing breast cancer recurrence risk are compelling and provide a strong justification for a randomized trial. Furthermore, similar to the aspirin data, data from the multiple randomized trials of statins for cardiovascular disease prevention/treatment should be pooled to evaluate for possible effects on cancer recurrence and mortality.

\section{Digoxin}

\section{Biological rationale/preclinical data}

Cardiac glycosides (the most widely used of which is digoxin) have also demonstrated anti-tumor effects in vitro, presumably through inhibition of $\mathrm{Na}^{+} \mathrm{K}^{+}$-ATPase. A variety of anti-tumor effects, including induction of apoptosis and inhibition of DNA topoisomerase II, have been observed in cell lines [18,37,38].

\section{Epidemiologic data}

Only one study (which has been published several times at varying times of follow-up) has evaluated the association between breast cancer recurrence and digoxin use. With 22 years of follow-up, breast cancer survivors who used digoxin had a lower rate of death $(6 \%, \mathrm{n}=32)$ from breast cancer than non-users $(34 \%, \mathrm{n}=143)$ [39]. To date, no other study on this topic has been published.

\section{Anti-diabetic medications \\ Biological rationale/preclinical data}

Although insulin, sulfonylureas, thiazolidinediones, and metformin all lower glucose levels and have been used successfully for diabetes treatment, they have different mechanisms of action and different associations with breast cancer survival. In terms of its anti-diabetic effect, metformin inhibits hepatic gluconeogenesis and improves insulin sensitivity in peripheral tissue [40]. The mechanism of action for thiazolidinediones (for example, rosiglitazone) is not completely understood, but they increase glucose utilization in adipose, muscle, and hepatic tissue, most likely by activating peroxisome proliferator-active receptors (PPARs). Both metformin and thiazolidinediones are associated with lower fasting insulin and C-peptide levels. Importantly, insulin is a known mitogen and can activate insulin-like growth factor 1 (IGF-1) receptors. Sulfonylureas stimulate the release of insulin from pancreatic beta cells. Higher circulating insulin has been associated with worse breast cancer mortality [41]. Since most of the studies that have evaluated the effect of anti-diabetic medications on cancer survival have compared recurrence risks across categories of drugs, all classes will be discussed concurrently [42].

\section{Epidemiologic data}

Because of the important biologic differences among the anti-diabetic medications, in addition to the overall search criteria, we limited our review to studies that reported results separately for types of anti-diabetic medication. In addition, we reviewed an intriguing study looking at pathologic complete response rates by antidiabetic medication use. Interestingly, the studies have been surprisingly consistent, showing a decreased risk of all-cancer mortality among diabetics who use metformin compared with those who use sulfonylureas or insulin. For a variety of reasons, these observational studies can be challenging to interpret. Diabetics have greater comorbidity and shorter life expectancy and so may get 
less aggressive cancer screening or treatment and may have a higher risk of adverse events from treatments [43]. In addition, the use of certain anti-diabetic drugs may be associated with certain prognostic factors. For example, metformin users tend to be younger than subjects who use other anti-diabetic medications. Analyses are further complicated by the fact that diabetic patients switch back and forth over time between mono-therapy and combined therapy.

The study with the largest number of breast cancer cases was a retrospective study of 112,408 subjects from the UK with a diagnosed solid tumor; 8,392 of the subjects had type II diabetes. Medication use was confirmed with pharmacy records. Among 25,575 breast cancer survivors (1,182 with type II diabetes), there was an increased risk of breast cancer mortality associated with having type II diabetes (unadjusted HR = 1.32, 95\% $\mathrm{CI}=1.17$ to 1.49 ). However, metformin use among cancer survivors was associated with a decreased risk of overall mortality (adjusted $\mathrm{HR}=0.85,95 \% \mathrm{CI}=0.78$ to 0.93) compared with non-diabetics. In contrast, diabetics who used sulfonylureas $(\mathrm{HR}=1.13,95 \% \mathrm{CI}=1.05$ to $1.21)$ or insulin ( $\mathrm{HR}=1.13,95 \% \mathrm{CI}=1.01$ to 1.27$)$ had an increased risk of mortality. These differences were not significant in analyses limited to breast cancer survivors [44]. In two companion studies, investigators from MD Anderson focused on outcomes among specific breast cancer subgroups. In the first study, they retrospectively reviewed 1,983 consecutive patients with stage II to IV HER2-positive breast cancer (154 diabetics) and found again that diabetes was associated with worse overall survival (adjusted $\mathrm{HR}=1.42,95 \% \mathrm{CI}=1.04$ to 1.94). However, in multivariate analyses, survival differed by anti-diabetic therapy, and insulin users had a shorter survival than diabetics who did not use insulin and nondiabetics. In contrast, diabetics who used metformin had significantly longer survival compared with diabetics who did not use metformin or non-diabetics. In multivariate analyses, both metformin use and thiazolidinedione use were associated with improved survival after adjustment for age, BMI, ER status, and use of insulin or insulin secretagogue therapy. In an analysis of competing risks among diabetic patients, metformin and thiazolidinodiones were associated with decreased breast cancerspecific mortality [42]. The second study focused on triple-negative breast cancer and included 1,448 women (including 63 diabetics on metformin and 67 diabetics not on metformin) with stage I to III triple-negative breast cancer treated with adjuvant chemotherapy between 1995 and 2007. In multivariate analyses, both diabetics not on metfomin and non-diabetics had a nonsignificant increased risk of distant metastases $(\mathrm{HR}=$ $1.63,95 \% \mathrm{CI}=0.87$ to 3.06 and $\mathrm{HR}=1.62,95 \% \mathrm{CI}=0.97$ to 2.71 , respectively) compared with diabetics on metformin. The study was limited by the small number of distant recurrences among the diabetics (18 among metformin users and 26 among non-metformin users) and limited data on metformin use, which were available for the adjuvant chemotherapy period only [46].

Among 2,529 breast cancer patients who received neoadjuvant chemotherapy for breast cancer at MD Anderson, the rate of pathologic complete response was higher in the metformin group $(24 \%, \mathrm{n}=68)$ compared with diabetics who did not take metformin $(8 \%, \mathrm{n}=87)$ and non-diabetics $(16 \%, \mathrm{n}=2,374)$ (overall $P$ for difference $=$ 0.02 ). Metformin was also independently predictive of the chances of pathologic complete response (adjusted odds ratio $=2.95,95 \% \mathrm{CI}=1.07$ to 8.17 ) after adjustment for age, diabetes, BMI, stage, grade, ER status, and treatment) [45].

The molecular basis for metformin's inhibition of cancer cell growth is not known but is hypothesized to be its ability to inhibit PI3-kinase/AKT/mammalian target to rapamycin (mTOR) signaling via activation of the LKB1/AMP-activated protein kinase (AMPK) pathway. Of all the medications presented in this review article, metformin is the only one that will have randomized trial data evaluating its effect on breast cancer recurrence within the near future. The National Cancer Institutes of Canada and US are enrolling subjects for a phase III study to evaluate the effect of metformin compared with placebo among women with higher-risk stage I and stage II or III breast cancer (NCIC MA32). The accrual of this study, which opened in April 2010 and is expected to close in 2016, is estimated to be 3,852 , and the results are eagerly awaited. Because of their effects on the PPAR pathway, ongoing phase I clinical trials are using a variety of thiazolidnediones in combination with chemotherapy for advanced solid tumors.

\section{Conclusions}

Substantial scientific evidence supports the hypothesis that several common and relatively safe drugs may reduce breast cancer mortality among breast cancer survivors by an amount that rivals the benefit of currently used therapies. In particular, the evidence is strongest for aspirin (approximately 50\% reduction), statins (approximately $25 \%$ reduction), and metformin (approximately $50 \%$ reduction).

We believe that randomized trials of aspirin, metformin, and statins are essential to move the field forward. Despite the compelling evidence presented in this review, it is based primarily on observational studies, which are subject to confounding. These drugs are generally safe, and their side effect profiles compare favorably with those of drugs used to treat cancer. However, we cannot estimate the overall risk-benefit ratio of these drugs without a randomized trial. For 
example, aspirin has a measurable risk of gastrointestinal [47] and central nervous system [48] bleeding, and there is a suggestion of hepatoxicity with metformin [49]. In addition, aspirin is not taken in a fixed dose; a randomized trial could help to establish the lowest effective dose.

If these findings are confirmed in randomized trials among breast cancer survivors, the public health impact would be immense. We estimate that, if aspirin is effective, using it to treat all patients with breast cancer in the US could potentially save 10,000 lives per year. In addition, if one considers the possible benefit in the developing world of an inexpensive, widely available medicine, the impact is truly staggering; an estimated 75,000 lives would potentially be saved each year.

In an era in which we struggle to contain health-care costs, the extra costs for patients with breast cancer in the US would be minimal. For developing countries, it could mean the difference between some adjuvant treatment and none. Whereas new cancer treatments typically benefit only patients in wealthy countries because of the costs, these drugs would be a breast cancer treatment available to every part of the world. The results of these trials could be truly transformative and change the treatment of breast cancer across the globe with what millions of people already have in their medicine cabinet.

Given the overwhelming weight of the biologic and observational data, randomized trials are the definitive way to assess the risk-benefit balance for breast cancer survivors. One such trial is under way for metformin. A similar trial for aspirin is definitely warranted, and possibly one for statins. We estimate that a trial of aspirin would require approximately 3,000 women with stage II or III breast cancer randomly assigned 1:1 and followed for 5 years and cost approximately \$15 million USD. However, because these drugs are generic and widely available, there is little industry incentive to support such studies. We propose that the cost is small given the potential benefit. Who will fill this need?

\section{Abbreviations}

$A C E$, angiotensin-converting enzyme; $A C E l$, angiotensin-converting enzyme inhibitor; ARB, angiotensin type I receptor blocker; BMI, body mass index; $\mathrm{Cl}$, confidence interval; COX, cyclo-oxygenase; ER, estrogen receptor; HR, hazard ratio; LACE, Life After Cancer Epidemiology; NSAID, non-steroidal antiinflammatory drug; PPAR, peroxisome proliferator-active receptor; RAAS, reninangiotensin-aldosterone system; RR, relative risk.

\section{Competing interests}

The authors declare that they have no competing interests.

\section{Author details}

'The Channing Division of Network Medicine, Department of Medicine, Brigham and Women's Hospital and Harvard Medical School, 181 Longwood Avenue, Boston, MA 02115, USA. 2Department of Epidemiology, Harvard School of Public Health, 677 Huntington Avenue, Boston, MA 02115, USA. ${ }^{3}$ Department of Medical Oncology, Dana Farber Cancer Institute, 450 Brookline Avenue, Boston, MA 02215, USA.
Published: 10 December 2012

\section{References}

1. Pereg $D$, Lishner M: Non-steroidal anti-inflammatory drugs for the prevention and treatment of cancer. J Intern Med 2005, 258:115-123.

2. Karmali RA, Welt S, Thaler HT, Lefevre F: Prostaglandins in breast cancer: relationship to disease stage and hormone status. Br J Cancer 1983, 48:689-696.

3. Leahy KM, Ornberg RL, Wang Y, Zweifel BS, Koki AT, Masferrer JL: Cyclooxygenase- 2 inhibition by celecoxib reduces proliferation and induces apoptosis in angiogenic endothelial cells in vivo. Cancer Res 2002, 62:625-631.

4. Tsujii M, DuBois RN: Alterations in cellular adhesion and apoptosis in epithelial cells overexpressing prostaglandin endoperoxide synthase 2 . Cell 1995, 83:493-501.

5. Terry MB, Gammon MD, Zhang FF, Tawfik H, Teitelbaum SL, Britton JA, Subbaramaiah K, Dannenberg AJ, Neugut Al: Association of frequency and duration of aspirin use and hormone receptor status with breast cancer risk. JAMA 2004, 291:2433-2440.

6. Gates MA, Tworoger SS, Eliassen AH, Missmer SA, Hankinson SE: Analgesic use and sex steroid hormone concentrations in postmenopausal women. Cancer Epidemiol Biomarkers Prev 2010, 19:1033-1041.

7. Holmes MD, Chen WY, Schnitt SJ, Collins L, Colditz GA, Hankinson SE, Tamimi RM: COX-2 expression predicts worse breast cancer prognosis and does not modify the association with aspirin. Breast Cancer Res Treat 2011, 130:657-662.

8. Dang CT, Dannenberg AJ, Subbaramaiah K, Dickler MN, Moasser MM, Seidman AD, D'Andrea GM, Theodoulou M, Panageas KS, Norton L, Hudis CA: Phase II study of celecoxib and trastuzumab in metastatic breast cancer patients who have progressed after prior trastuzumab-based treatments. Clin Cancer Res 2004, 10:4062-4067.

9. Gay LJ, Felding-Habermann B: Contribution of platelets to tumour metastasis. Nat Rev Cancer 2011, 11:123-134.

10. Kwan ML, Habel LA, Slattery ML, Caan B: NSAIDs and breast cancer recurrence in a prospective cohort study. Cancer Causes Control 2007, 18:613-620.

11. Blair CK, Sweeney C, Anderson KE, Folsom AR: NSAID use and survival after breast cancer diagnosis in post-menopausal women. Breast Cancer Res Treat 2007, 101:191-197.

12. Holmes MD, Chen WY, Li L, Hertzmark E, Spiegelman D, Hankinson SE: Aspirin intake and survival after breast cancer. J Clin Oncol 2010, 28:1467-1472.

13. Howe LR, Lippman SM: Modulation of breast cancer risk by nonsteroidal anti-inflammatory drugs. J Natl Cancer Inst 2008, 100:1420-1423.

14. Li Y, Brasky TM, Nie J, Ambrosone CB, McCann SE, Shields PG, Trevisan M, Edge $S B$, Freudenheim JL: Use of nonsteroidal anti-inflammatory drugs and survival following breast cancer diagnosis. Cancer Epidemiol Biomarkers Prev 2012, 21:239-242.

15. Rothwell PM, Wilson M, Price JF, Belch JF, Meade TW, Mehta Z: Effect of daily aspirin on risk of cancer metastasis: a study of incident cancers during randomised controlled trials. Lancet 2012, 379:1591-1601.

16. Algra AM, Rothwell PM: Effects of regular aspirin on long-term cancer incidence and metastasis: a systematic comparison of evidence from observational studies versus randomised trials. Lancet Oncol 2012 13:518-527.

17. Antoni MH, Lutgendorf SK, Cole SW, Dhabhar FS, Sephton SE, McDonald PG, Stefanek M, Sood AK: The influence of bio-behavioural factors on tumour biology: pathways and mechanisms. Nat Rev Cancer 2006, 6:240-248.

18. Vaklavas C, Chatzizisis YS, Tsimberidou AM: Common cardiovascular medications in cancer therapeutics. Pharmacol Ther 2011, 130:177-190.

19. Sloan EK, Priceman SJ, Cox BF, Yu S, Pimentel MA, Tangkanangnukul V, Arevalo JM, Morizono K, Karanikolas BD, Wu L, Sood AK, Cole SW: The sympathetic nervous system induces a metastatic switch in primary breast cancer. Cancer Res 2010, 70:7042-7052.

20. Vandewalle B, Revillion F, Lefebvre J: Functional beta-adrenergic receptors in breast cancer cells. J Cancer Res Clin Oncol 1990, 116:303-306.

21. Powe DG, Voss MJ, Zanker KS, Habashy HO, Green AR, Ellis IO, Entschladen F: Beta-blocker drug therapy reduces secondary cancer formation in breast cancer and improves cancer specific survival. Oncotarget 2010, 1:628-638.

22. Melhem-Bertrandt A, Chavez-Macgregor M, Lei X, Brown EN, Lee RT, MericBernstam F, Sood AK, Conzen SD, Hortobagyi GN, Gonzalez-Angulo AM: Beta-blocker use is associated with improved relapse-free survival in 
patients with triple-negative breast cancer. J Clin Oncol 2011, 29:2645-2652.

23. Ganz PA, Habel LA, Weltzien EK, Caan BJ, Cole SW: Examining the influence of beta blockers and ACE inhibitors on the risk for breast cancer recurrence: results from the LACE cohort. Breast Cancer Res Treat 2011, 129:549-556.

24. Barron TI, Connolly RM, Sharp L, Bennett K, Visvanathan K: Beta blockers and breast cancer mortality: a population- based study. J Clin Oncol 2011, 29:2635-2644

25. Koh WP, Yuan JM, Sun CL, van den Berg D, Seow A, Lee HP, Yu MC: Angiotensin l-converting enzyme (ACE) gene polymorphism and breast cancer risk among Chinese women in Singapore. Cancer Res 2003, 63:573-578.

26. Koh WP, Yuan JM, Van Den Berg D, Lee HP, Yu MC: Polymorphisms in angiotensin II type 1 receptor and angiotensin I-converting enzyme genes and breast cancer risk among Chinese women in Singapore. Carcinogenesis 2005, 26:459-464.

27. Herr D, Rodewald M, Fraser HM, Hack G, Konrad R, Kreienberg R, Wulff C: Potential role of Renin-Angiotensin-system for tumor angiogenesis in receptor negative breast cancer. Gynecol Oncol 2008, 109:418-425.

28. Greco S, Muscella A, Elia MG, Salvatore P, Storelli C, Mazzotta A, Manca C, Marsigliante S: Angiotensin II activates extracellular signal regulated kinases via protein kinase $C$ and epidermal growth factor receptor in breast cancer cells. J Cell Physiol 2003, 196:370-377.

29. Zhao Y, Chen X, Cai L, Yang Y, Sui G, Fu S: Angiotensin II/angiotensin II type I receptor (AT1R) signaling promotes MCF-7 breast cancer cells survival via PI3-kinase/Akt pathway. J Cell Physio/ 2010, 225:168-173.

30. Chae YK, Valsecchi ME, Kim J, Bianchi AL, Khemasuwan D, Desai A, Tester W: Reduced risk of breast cancer recurrence in patients using ACE inhibitors, ARBs, and/or statins. Cancer Invest 2011, 29:585-593.

31. Khanzada UK, Pardo OE, Meier C, Downward J, SeckI MJ, Arcaro A: Potent inhibition of small-cell lung cancer cell growth by simvastatin reveals selective functions of Ras isoforms in growth factor signalling. Oncogene 2006, 25:877-887.

32. Lin YC, Lin JH, Chou CW, Chang YF, Yeh SH, Chen CC: Statins increase p21 through inhibition of histone deacetylase activity and release of promoter-associated HDAC1/2. Cancer Res 2008, 68:2375-2383.

33. Kwan ML, Habel LA, Flick ED, Quesenberry CP, Caan B: Post-diagnosis statin use and breast cancer recurrence in a prospective cohort study of early stage breast cancer survivors. Breast Cancer Res Treat 2008, 109:573-579.

34. Ahern TP, Pedersen L, Tarp M, Cronin-Fenton DP, Garne JP, Silliman RA, Sorensen HT, Lash TL: Statin prescriptions and breast cancer recurrence risk: a Danish nationwide prospective cohort study. J Nat/ Cancer Inst 2011 103:1461-1468.

35. Jackevicius CA, Chou MM, Ross JS, Shah ND, Krumholz HM: Generic atorvastatin and health care costs. N Eng/ J Med 2012, 366:201-204

36. Duncan RE, El-Sohemy A, Archer MC: Statins and cancer development. Cancer Epidemiol Biomarkers Prev 2005, 14:1897-1898.
37. Frese S, Frese-Schaper M, Andres AC, Miescher D, Zumkehr B, Schmid RA: Cardiac glycosides initiate Apo2L/TRAIL-induced apoptosis in non-small cell lung cancer cells by up-regulation of death receptors 4 and 5 . Cancer Res 2006, 66:5867-5874.

38. Bielawski K, Winnicka K, Bielawska A: Inhibition of DNA topoisomerases I and II, and growth inhibition of breast cancer MCF-7 cells by ouabain, digoxin and proscillaridin A. Biol Pharm Bull 2006, 29:1493-1497.

39. Stenkvist B: Is digitalis a therapy for breast carcinoma? Oncol Rep 1999, 6:493-496

40. Decensi A, Puntoni M, Goodwin P, Cazzaniga M, Gennari A, Bonanni B, Gandini S: Metformin and cancer risk in diabetic patients: a systematic review and meta-analysis. Cancer Prev Res (Phila Pa) 2010, 3:1451-1461.

41. Goodwin PJ, Ennis M, Pritchard KI, Trudeau ME, Koo J, Madarnas Y, HartwickW, Hoffman B, Hood N: Fasting insulin and outcome in early-stage breast cancer: results of a prospective cohort study. J Clin Oncol 2002, 20:42-51.

42. He X, Esteva FJ, Ensor J, Hortobagyi GN, Lee MH, Yeung SC: Metformin and thiazolidinediones are associated with improved breast cancer-specific survival of diabetic women with HER2+ breast cancer. Ann Oncol 2012 23:1771-1780

43. Peairs KS, Barone BB, Snyder CF, Yeh HC, Stein KB, Derr RL, Brancati FL, Wolff AC: Diabetes mellitus and breast cancer outcomes: a systematic review and meta-analysis. J Clin Oncol 2011, 29:40-46.

44. Currie CJ, Poole CD, Jenkins-Jones S, Gale EA, Johnson JA, Morgan CL: Mortality after incident cancer in people with and without type 2 diabetes: impact of metformin on survival. Diabetes Care 2012, 35:299-304.

45. Jiralerspong S, Palla SL, Giordano SH, Meric-Bernstam F, Liedtke C, Barnett CM, Hsu L, Hung MC, Hortobagyi GN, Gonzalez-Angulo AM: Metformin and pathologic complete responses to neoadjuvant chemotherapy in diabetic patients with breast cancer. J Clin Oncol 2009, 27:3297-3302

46. Bayraktar S, Hernadez-Aya LF, Lei X, Meric-Bernstam F, Litton JK, Hsu L, Hortobagyi GN, Gonzalez-Angulo AM: Effect of metformin on survival outcomes in diabetic patients with triple receptor-negative breast cancer. Cancer 2012, 118:1202-1211.

47. Hernandez-Diaz S, Garcia Rodriguez LA: Cardioprotective aspirin users and their excess risk of upper gastrointestinal complications. BMC Med 2006 $4: 22$.

48. He J, Whelton PK, Vu B, Klag MJ: Aspirin and risk of hemorrhagic stroke: a meta-analysis of randomized controlled trials. JAMA 1998, 280:1930-1935.

49. Hashmi T: Probable hepatotoxicity associated with the use of metformin in type 2 diabetes. BMJ Case Rep 2011, 2011.

doi:10.1186/bcr3336

Cite this article as: Holmes MD, Chen WY: Hiding in plain view: the potential for commonly used drugs to reduce breast cancer mortality. Breast Cancer Research 2012, 14:216. 